\title{
The maximum relative growth rate of common UK plant species is positively associated with their global invasiveness
}

\author{
Wayne Dawson*, Markus Fischer and Mark van Kleunen
}

\begin{abstract}
Aim An emerging consensus in invasion ecology is that faster-growing alien plant species tend to be more invasive than slower-growing species. However, phylogenetic non-independence and the precision of growth-rate measures often remain unaccounted for in comparative studies. We tested whether global invasiveness was related to mean and maximum relative growth rate of 105 plant species (101 native and 4 introduced) commonly occurring in the UK.
\end{abstract}

Location Global.

Methods We combined a unique experimental dataset of relative growth rates (RGR) measured under standardized experimental conditions for plant species that occur widely in the UK with our global measures of invasiveness, which were the number of references in the Global Compendium of Weeds (GCW) and the number of world regions invaded. We weighted mean RGR measures per species by including variances of RGR in our analyses, and we also conducted analyses with and without phylogenetic structure, to account for potential phylogenetic nonindependence in RGR.

Results We found a positive association between global invasiveness and maximum RGR. In addition, this association was not confounded by phylogenetic correlation, or by species seed mass.

Main conclusions The results from this study suggest that faster-growing species are more widespread at a global scale, adding support to other studies that suggest faster-growing alien plant species tend to be more invasive in the introduced range.

Keywords

Exotic, introduced, invasion success, life history, plant growth, weeds.

\section{INTRODUCTION}

Despite the explosion in invasion ecology literature in recent years (Pyšek et al., 2008), there are frustratingly few generalizations that can be made regarding the determinants of invasiveness among alien species and across studies (Hayes \& Barry, 2008). One emerging exception among alien plants is that invasive species tend to be faster growing than non-invasive species (Rejmánek, 2005; Grotkopp \& Rejmánek, 2007) or have traits correlated with faster relative growth rates compared with native and non-invasive alien species such as large specific leaf area and high foliar nutrient concentrations (Hamilton et al., 2005; Rejmánek, 2005; Leishman et al., 2007; Peñuelas et al., 2010) and small seed size (Rejmánek \& Richardson, 1996; Grotkopp et al., 2002; Richardson \& Rejmánek, 2004). Plant growth rate repre- sents an important axis of life-history variation among species, with faster-growing species tending to be shade-intolerant colonizers of open habitats, with fewer anti-herbivore defences, shorter life spans, greater resource capture ability (Wright et al., 2004) and higher reproductive output than slower-growing species (Rose et al., 2009). Therefore, plant growth rate has received much interest as a potential key trait that might be related to invasion success.

However, not all studies show support for this relationship (Bellingham et al., 2004; Martin et al., 2009), and studies have typically been limited to fewer than 40 species (Grotkopp et al., 2002; Bellingham et al., 2004; Grotkopp \& Rejmánek, 2007). In addition, generalization across studies and species can be troublesome if plants are grown under different environmental conditions, which will affect relative growth rates. For example, 
maximum relative growth rate measured under optimal conditions after germination might provide a better measure of growth potential than mean relative growth rate over time (Turnbull et al., 2008). Furthermore, relatively few invasion studies investigating differences in growth rates have attempted to control for phylogenetic or taxonomic non-independence (but see Grotkopp et al., 2002; Bellingham et al., 2004). Finally, many invasion studies that suggest that certain trait values (including growth rates) are indicative of invasiveness only use mean trait values, which has the result of treating trait values of all species as if they do not vary within species, and all species are given equal weighting in analyses. This is clearly unrealistic, as all traits are likely to vary among individuals due to genotypic and environmental variation and measurement error. Neglecting intra-specific variability in trait values and treating all species as equal could lead to spurious conclusions about the importance of those traits in conferring invasiveness.

In this study, we aimed to address these issues by using a unique dataset of Grime \& Hunt (1975) on mean and maximum relative growth rates measured soon after germination for 105 species commonly occurring in the UK. Most of these species are native and widespread in the UK and Europe occurring in multiple habitats, but especially grasslands and more open habitats (Grime \& Hunt, 1975). Given widespread European colonial activity from the 17 th to 20 th centuries, it is likely that these species have been widely dispersed globally in the past. This dataset is valuable, as all plant species were grown under controlled experimental conditions, thus minimizing the problem of inconsistencies in growth and measurement methods among species. In addition, standard errors and sample sizes have been included with mean relative growth rate values per species, which allows variation (and therefore precision) in relative growth rate measurements to be included in analyses. In addition, we make use of a constructed phylogeny for this dataset in order to account for potential phylogenetic non-independence when assessing the relationship between species relative growth rates and invasiveness. We combine this dataset with two global measures of species invasiveness outside their native ranges: (1) the number of references in the Global Compendium of Weeds (GCW) (Randall, 2002) describing a species as invasive; and (2) the number of global regions where the species is recorded (maximum of 10; Table 1), also derived from the GCW. Whilst disentangling global introduction effort from perceived invasiveness is difficult, this compendium has been used elsewhere (van Kleunen et al., 2007; Pyšek et al., 2009; Schlaepfer et al., 2010) and is currently the most exhaustive, world-wide compilation of 284 weed and invasive plant list references. Thus it is likely to give the best indication of how invasive a species is at a global scale.

\section{METHODS}

\section{Data}

The relative growth rate (RGR) data used came from a comparative study conducted by Grime \& Hunt (1975) involving 132 species, many of which co-occur in a number of UK plant communities. All species were germinated and grown for 5 weeks under standardized experimental conditions, with high nutrient, light and water availability, and with four harvests in weeks $2-5$. All species and all four harvests were replicated five times (Grime \& Hunt, 1975). Two measures of RGR were calculated. Firsty, mean RGR per week was calculated as the difference in the natural log of the weight between week 5 and week 2 , divided by the number of weeks. Secondly, the maximum RGR was obtained by taking the highest value of RGR achieved throughout the experiment between any two harvests, and calculating the average from the five replicates per species (Grime \& Hunt,

Table 1 The 10 regions where plant species in this study have been recorded as casual aliens, naturalized or as invasive according to references in the Global Compendium of Weeds (Randall, 2002).

\begin{tabular}{llr}
\hline Region & Countries included & $\begin{array}{c}\text { Total number of } \\
\text { references cited per region }\end{array}$ \\
\hline $\begin{array}{l}\text { North America } \\
\text { South America }\end{array}$ & USA, Canada & 59 \\
& Argentina, Brazil, Chile, Suriname, Guyana, French Guiana, Venezuela, & 11 \\
Central America & Peru, (South America) & 5 \\
Africa & Cuba, Mexico, Puerto Rico, (Central America) & 13 \\
Pacific & South Africa, Sudan, East Africa, Egypt, Ethiopia, Zimbabwe, Zambia & 3 \\
Australasia & Galapagos, Pacific islands, Pohnpei & 31 \\
Middle East & Australia, New Zealand & 2 \\
South Asia & Syria, (Middle East) & 2 \\
East Asia & Bangladesh, Pakistan & 10 \\
Southeast Asia & China, Japan, Mongolia, Taiwan, (Asia) & 5
\end{tabular}

Included are the countries within regions corresponding to references, and the total number of references for each region cited for species in this study. In brackets are the regions themselves that are represented by references covering multiple unspecified countries in the region. Note that individual references are mutually exclusive at the regional level, such that for example, a reference for 'Asia' is only counted once as representing 'East Asia' (the largest Asian region), and is not counted again for South Asia or Southeast Asia. 
1975). It was recognized that these values for maximum RGR represented approximations of the true maximum growth potential of plants at this early phase of growth after germination (Grime \& Hunt, 1975). For the current study, average mean and maximum RGR per species were extracted along with associated standard errors. These standard errors and the number of replicates were then used to calculate variances of RGR measures for each species.

Our first measure of invasiveness in this study was the number of references recording a species as invasive or weedy in the GCW (Randall, 2002). To prevent the inclusion of species that may only be considered weeds in their native ranges, species were only included if cited as 'naturalized', 'environmental weeds', 'sleeper weeds', 'casual aliens' or/and 'cultivation escapes' (Randall, 2002). Because some global regions may have a greater bias in recording alien invasive species (such as North America and Australasia; see Table 1), the number of GCW references may not clearly indicate the global level of species spread. Therefore, we calculated a second measure of invasiveness which was the number of global regions containing a GCW reference, obtained from countries and regions listed per reference in the GCW (Randall, 2002). The total number of 10 regions included both continents and continental regions (Table 1). This second measure avoids the double counting of invaded regions inherent in counting the number of references.

We focused our study on 105 terrestrial herbaceous forb and graminoid species with RGR measures (Grime \& Hunt, 1975) which were present in the GCW. We chose to exclude woody species from the original study, as they were poorly represented (17 species). The species considered are native and common to the UK, except for four naturalized alien species: Senecio squalidus, Lycopersicon esculentum, Matricaria discoidea and Fallopia convolvulus. In total, there were 36 grass species ( 5 annual and 31 perennial), 65 herbaceous dicot species (14 annual and 51 biennial/perennial), 2 Cyperaceae species (perennial) and 3 Juncaceae species (perennial; see Appendix $S 1$ in the Supporting Information for a species list). Except for $S$. squalidus, $L$. esculentum and $M$. discoidea, all species are native to Europe, and as such only GCW references describing these three species as at least casual aliens or escaping cultivation in Europe were included; all other European references were excluded. This restricted the species considered to those that we knew had definitely been introduced outside their native range and were also capable of producing offspring in uncultivated conditions. For these species, we checked for congruence between the number of references in the GCW and an updated global list of invasive species (Weber, 2003). We found that species in this invasive list had a median of $41 \mathrm{GCW}$ references $(n=31)$ and five regions, while species not on the list had a median of 10.5 references $(n=74)$ and three regions. This congruence indicates that our measures of global invasiveness were robust. As seed mass has also often been shown to correlate both with alien plant invasiveness (Rejmánek, 2005) and plant growth rate (Turnbull et al., 2008), we aimed to account for the potential confounding effect of seed mass on invasiveness-RGR relationships. We obtained seed mass data ( $\mathrm{mg}$ dry weight) for all 105 species from the Seed Information Database (Liu et al., 2008) and the BIOLFLOR plant database (Klotz et al., 2002).

\section{Data analysis}

We took a Bayesian approach to analysing the data with generalized linear mixed models, using the $\mathrm{R}$ package $\mathrm{MCMCglmm}$ (Hadfield, 2010). This package allows models to be fitted to data that include more than one explanatory variable and the variance of the response variable per data point, and it also allows phylogenies to be included (Hadfield, 2010). The resulting analysis effectively resembles a phylogenetic meta-analysis (Lajeunesse, 2009) by integrating the variance (and therefore the precision) of the response variable values for individual species with a matrix of phylogenetic relatedness among species. In our analyses, relative growth rate measures were the response variables (with associated variances), and our measures of invasiveness were explanatory variables in separate analyses for each RGR measure. We treated RGR measures instead of global invasiveness measures as response variables, because this allowed the variance of RGR measures to be incorporated into analyses.

A phylogeny (see Appendix S2) was also incorporated into our analyses. The topology of the phylogeny was obtained via the online program Phylomatrc (Webb \& Donoghue, 2005), using a constructed angiosperm phylogeny (Stevens, 2001 onwards) as the base tree. Polytomies within families were resolved as far as possible using published phylogenies obtained through molecular analysis, in particular for the Asteraceae (Goertzen et al., 2003), Caryophyllaceae (Fior et al., 2006), Brassicaceae (Bailey et al., 2006), Fabaceae (Wojciechowski et al., 2004), Polygonaceae (Lamb Frye \& Kron, 2003) and Poaceae (Bouchenak-Khelladi et al., 2008). Branch length estimates were obtained and included in phylogenetic trees by calibrating the topology according to 'known' fixed ages of the tree root and divergence at a number of tree nodes from fossil records (Stevens, 2001 onwards; Wilkström et al., 2001), using the program Pнy Locom version 4.1 (Webb et al., 2008). Whilst this calibration technique may not yield phylogenies with accurate branch length estimates, it is likely to provide the 'best guess' of the true phylogeny and relatedness among species according to available fossil evidence (Webb et al., 2008).

Models were initially run with the invasion measure included as the sole explanatory variable, and the response variable was assumed to have a Gaussian error distribution. Some species included had an annual or biennial life history (i.e. monocarpic) whilst others were perennial (polycarpic), and we might expect annual and biennial species to have faster relative growth rates than perennial species (Grime \& Hunt, 1975). We tested life history alone as an explanatory variable to account for this, and we also checked for interactions between number of GCW references or regions invaded and life history. The few biennial species $(n=5)$ in the dataset were grouped with annuals $(n=19)$ to form one monocarpic group $(n=24)$ and one polycarpic group $(n=81)$. Finally, in order to account for seed mass, we added log-transformed seed mass as a covariate in models with the two global invasion measures. We also tested for a relation- 
ship between seed mass as the dependent variable and invasion measures as the independent variable. Each model was run for 50,000 Markov chain Monte Carlo (MCMC) simulation iterations, with a burn-in of 25,000 iterations. Uninformative prior distributions were used for parameters, with mean of 0 and variance of $1 \times 10^{10}$. In prior specification, residual variancecovariance matrices were fixed to $l$, as we assumed that we knew the measurement error variances of RGR (Hadfield, 2010). We checked for convergence of model parameter estimates by inspecting trace plots of the MCMC iterations, and by using the Raftery-Lewis diagnostic. We chose a thinning interval of 10 iterations, which resulted in 2500 posterior distribution samples of model parameter estimates, from which mean parameter estimates were calculated, and $95 \%$ credible intervals were constructed. Parameters with credible intervals including zero were considered not significant.

All analyses were conducted both with no phylogenetic structure (i.e. assuming that all species are equally related to one another), and with the full phylogeny included. The models without phylogenetic structure were effectively random effects meta-analyses, with species included as random effects (Hadfield, 2010). The deviance information criterion (DIC) was calculated and compared to check if inclusion of phylogeny improved model fit. As with the Akaike information criterion, a lower DIC value indicates a better model, with differences of $>5$ indicating that one model is clearly better (Spiegelhalter $e t$ al., 2002; Bolker, 2008). Finally, we also checked if monocarpic and polycarpic species differed in their number of GCW references and the number of regions invaded, using generalized linear models with quasi-Poisson and Poisson error distributions, respectively. All analyses were conducted using the program $R$, version 2.9.1 (R Development Core Team, 2009).

\section{RESULTS}

Mean RGR values ranged from 0.5 to $2.2 \mathrm{~g} \mathrm{~g}^{-1}$ week $\mathrm{k}^{-1}$ across all species with a mean of $1.2 \mathrm{~g} \mathrm{~g}^{-1}$ week $^{-1}$, whereas maximum RGR ranged from 0.5 to $2.7 \mathrm{~g} \mathrm{~g}^{-1}$ week $\mathrm{k}^{-1}$ with a mean of $1.3 \mathrm{~g} \mathrm{~g}^{-1}$ week $^{-1}$. The maximum number of GCW references was 84 and the mean was 24.2 , whereas the maximum number of regions invaded was 9 , with a mean of 3.6 regions.

Mean RGR was neither strongly positively associated with the number of GCW references nor with the number of regions invaded, with $95 \%$ credible intervals encompassing zero, both with and without phylogenetic structure included (Table 2). However, maximum RGR was positively related to both the number of GCW references and regions invaded, with 95\% credible intervals not including zero (Table 2), indicating that faster-growing species in the near-exponential growth phase were considered globally more invasive (Fig. 1). In addition, the significant relationships between invasion measures and maximum RGR held when species' seed mass was included as a covariate, and seed mass was not significantly related to either RGR measure (Table 3 ). Seed mass was also not related to the number of GCW references per species $[\beta=$ $1.395,95 \%$ credible interval $(\mathrm{Cl})=(-1.418,4.174)]$ or the number of regions invaded $[\beta=0.112,95 \% \mathrm{Cl}=(-0.150$, 0.373)].

When life cycle (monocarpic/polycarpic) was considered alone as an explanatory variable, there was no significant difference in growth rate between monocarpic and polycarpic species (Table 2). There was also no significant interaction between life cycle and the number of GCW references, or regions invaded (Appendix S3). The number of GCW references was significantly lower for polycarpic species than monocarpic species $[\beta=-0.519,95 \% \mathrm{CI}=(-0.839,-0.187)$, quasi- $\left.F_{1,103}=9.17, P=0.003\right]$. Monocarpic species had a mean of $35.3 \pm 4.2 \mathrm{GCW}$ references (mean $\pm \mathrm{SEM}$ ), whereas polycarpic species had a mean of $21 \pm 2.1$ GCW references. Polycarpic species were also invading significantly fewer regions than monocarpic species $[\beta=-0.295,95 \% \mathrm{CI}=(-0.513$, $\left.-0.069), \chi_{1,103}^{2}=6.47, P=0.01\right]$, with a mean of $3.4 \pm 0.2$ regions invaded by polycarpic species compared with $4.5 \pm 0.4$ regions by monocarpic species.

Table 2 Mean parameter estimates and $95 \%$ credible intervals $(\mathrm{CI})$ describing the relationship between relative growth rate (RGR; $\mathrm{g} \mathrm{g}^{-1}$ week $^{-1}$ ) and number of Global Compendium of Weeds (GCW) references, number of regions invaded, and life cycle with and without including phylogeny.

\begin{tabular}{|c|c|c|c|c|}
\hline \multirow[b]{2}{*}{ Model } & \multicolumn{2}{|l|}{ No phylogeny } & \multicolumn{2}{|l|}{ With phylogeny } \\
\hline & Parameter estimate $(95 \% \mathrm{CI})$ & DIC & Parameter estimate $(95 \% \mathrm{CI})$ & DIC \\
\hline \multicolumn{5}{|l|}{ Mean RGR } \\
\hline GCW references & $0.007(-0.003,0.018)$ & 229.2 & $0.005(-0.009,0.020)$ & 294.1 \\
\hline GCW regions & $0.078(-0.029,0.182)$ & 229.0 & $0.060(-0.084,0.211)$ & 294.1 \\
\hline Polycarpic & $-0.089(-0.542,0.414)$ & 230.7 & $-0.021(-0.734,0.641)$ & 294.6 \\
\hline \multicolumn{5}{|l|}{ Maximum RGR } \\
\hline GCW references & $0.011(0.001,0.022)$ & 238.7 & $0.008(-0.006,0.023)$ & 299.2 \\
\hline GCW regions & $0.110(0.009,0.222)$ & 238.9 & $0.083(-0.067,0.226)$ & 298.7 \\
\hline Polycarpic & $-0.355(-0.867,0.111)$ & 240.8 & $-0.269(-0.997,0.415)$ & 299.3 \\
\hline
\end{tabular}

Polycarpic life-cycle parameter estimates are relative to monocarpic species. Significant parameters are in bold. Deviance information criterion values (DIC) are also provided. 

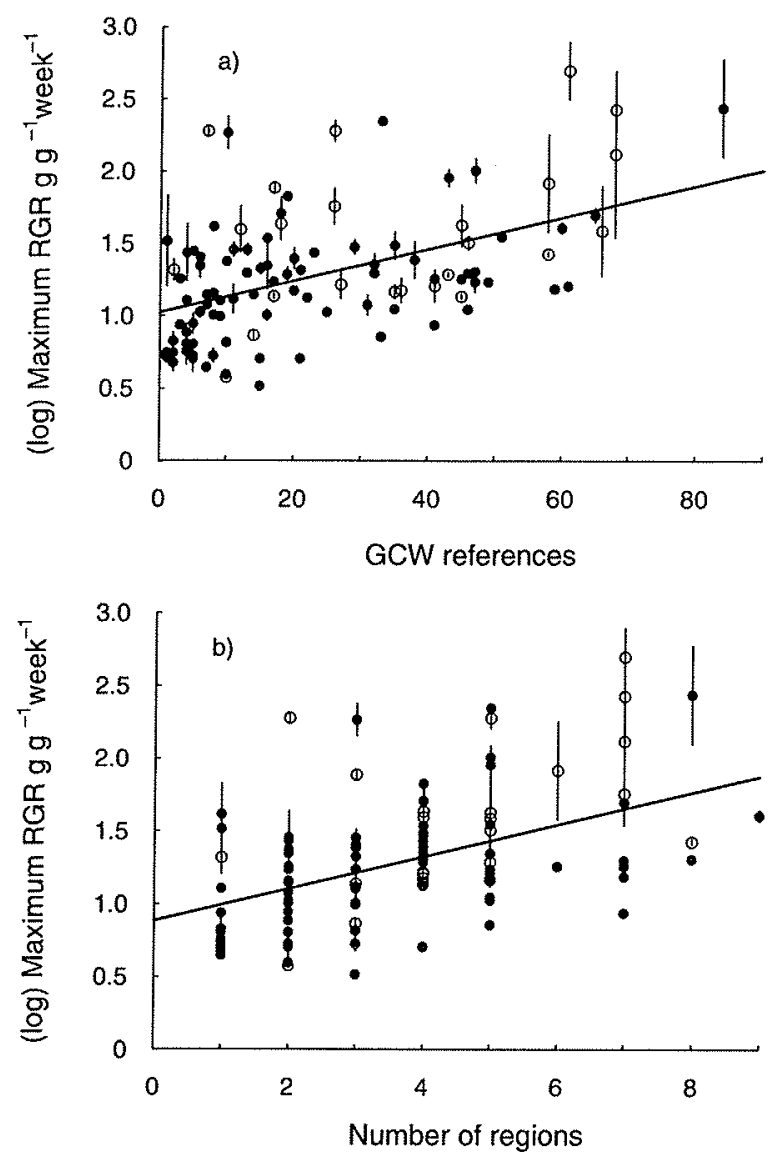

Figure 1 Average maximum relative growth rate (RGR) per week (natural log-transformed) in relation to (a) the number of references in the Global Compendium of Weeds (GCW; Randall, 2002) and (b) the number of regions recorded for 105 herbaceous plant species. Error bars represent \pm variance of RGR per species $(n=5)$. Filled circles, polycarpic species; open circles, monocarpic species. Fitted lines are from models including only number of GCW references (a) or number of regions (b) as a parameter and no phylogenetic structure.

Whilst the significance of the relationship between maximum RGR and both measures of invasiveness was reduced when phylogenetic structure was included in the model, the direction of the trends remained for both mean RGR and maximum RGR (Table 2). Moreover, DIC values were much higher in all models (including those inspecting life cycle) that included phylogeny, compared with those with no phylogenetic structure (Table 2). This increase in DIC suggested that inclusion of phylogenetic structure did not improve model fit, and that the relationships between RGR measures and global invasiveness measures were not confounded by phylogenetic correlation.

\section{DISCUSSION}

The results from our study lend support to the general assumption that faster-growing species tend to be more invasive at a global scale. Species that were faster growing at the early nearexponential phase of growth post-germination were listed in a greater number of invasion references included in the GCW. Inclusion of phylogenetic structure did not improve model fit, and did not change the positive trend between growth rates and invasiveness, indicating that the positive relationship found was not an artefact produced by a phylogenetic correlation of both growth rate and invasiveness. We also found that species with a higher maximum RGR had invaded a greater number of global regions. In addition, whilst seed mass is arguably a correlate of plant growth rates (Turnbull et al., 2008), the relationships between growth rate and our invasion measures were not confounded by seed mass, and invasion measures were not related to seed mass.

Whilst the measures of invasiveness used here may be considered coarse, the GCW is arguably the most exhaustive and globally encompassing list of alien invasive plants currently available, giving us the best indication of global spread by invasive plant species which corresponds well with other, less comprehensive, lists (Weber, 2003). Thus, our study demonstrates that invasiveness at a global scale can be related to how fast species can grow under optimal conditions after germination.

One caveat of most studies comparing species of differing invasiveness is the difficulty in disentangling introduction effort from intrinsic invasiveness and associated traits such as growth rate (Bellingham et al., 2004; Martin et al., 2009). Colonization pressure (the number of species introduced to a region) and propagule pressure (the number of individuals introduced per species) are difficult to fully quantify (but see Gravuer et al., 2008), despite recognition that they will influence the frequency and identity of introduced species that establish and the traits that are then considered successful (Lockwood et al., 2009). However, the species used in this study are common in their native range and are thus more likely to have been widely introduced to new ranges; only 10 of the 132 species in the original study (Grime \& Hunt, 1975) do not appear in the GCW (and these were excluded from our analyses), indicating that the remainder have been introduced at least once to a region outside of their native range. In addition, the vast majority of species in this study ( $n=103$ ) were listed in the GCW as at least naturalized in the introduced range, suggesting that propagule pressure has been sufficient to allow self-perpetuating populations of most species to establish in at least one introduced region. Despite recognition of the role of propagule pressure in invasions, it is still unclear at which phase and at which spatial scale of invasion it is most important (Dietz \& Edwards, 2006; Lockwood et al., 2009).

It has been suggested that the prevalence of faster growth rates among invasive alien plants could be an artefact of greater introduction bias of faster-growing commercially valuable species and varieties (Martin et al., 2009). One approach to check for global introduction bias in favour of faster-growing species would be to compare growth rates of species that do not occur in the GCW with those that do (van Kleunen et al., 2010a). Unfortunately, we were unable to adequately check for this bias using the original dataset (Grime \& Hunt, 1975), as only 10 herba- 
Table 3 Mean parameter estimates and $95 \%$ credible intervals $(\mathrm{Cl})$ describing the relationship between relative growth rate ( $R G R ; \mathrm{g} \mathrm{g}^{-1}$ week $^{-1}$ ) and number of Global Compendium of Weeds (GCW) references and number of regions invaded, with seed mass (log-transformed dry weight) added as a covariate.

\begin{tabular}{|c|c|c|c|c|}
\hline \multirow[b]{2}{*}{ Model } & \multicolumn{2}{|l|}{ No phylogeny } & \multicolumn{2}{|l|}{ With phylogeny } \\
\hline & Parameter estimate $(95 \% \mathrm{CI})$ & DIC & Parameter estimate $(95 \% \mathrm{CI})$ & DIC \\
\hline \multicolumn{5}{|c|}{ Mean RGR: GCW references + seed mass } \\
\hline GCW references & $0.074(-0.018,0.184)$ & 229.9 & $0.006(-0.008,0.020)$ & 288.4 \\
\hline Seed mass & $-0.066(-0.211,0.093)$ & & $-0.068(-0.299,0.136)$ & \\
\hline \multicolumn{5}{|c|}{ Mean RGR: GCW regions + seed mass } \\
\hline GCW regions & $0.083(-0.021,0.188)$ & 229.8 & $0.060(-0.079,0.199)$ & 288.4 \\
\hline Seed mass & $-0.084(-0.214,0.085)$ & & $-0.064(-0.299,0.139)$ & \\
\hline \multicolumn{5}{|c|}{ Maximum RGR: GCW references + seed mass } \\
\hline GCW references & $0.011(0.001,0.022)$ & 240.5 & $0.008(-0.007,0.022)$ & 293.5 \\
\hline Seed mass & $-0.024(-0.180,0.125)$ & & $-0.008(-0.228,0.215$ & \\
\hline \multicolumn{5}{|c|}{ Maximum RGR: GCW regions + seed mass } \\
\hline GCW regions & $0.114(0.002,0.213)$ & 240.7 & $0.082(-0.075,0.212)$ & 293.5 \\
\hline Seed mass & $-0.026(-0.182,0.130)$ & & $-0.004(-0.224,0.221)$ & \\
\hline
\end{tabular}

Model estimates are shown with and without including phylogenetic information. Significant parameters are in bold. Deviance information criterion values (DIC) are also provided.

ceous species were not recorded in the GCW. We also found that monocarpic species had a greater number of GCW references and were invading more regions on average than polycarpic species. This could be because annuals and biennials are more likely to be widely introduced and disseminated unintentionally as ruderal and agricultural weeds (Hulme, 2005) or because of reporting bias for annual and biennial weed species of anthropogenic habitats. Moreover, while several recent studies have found that invasive alien species are faster growing or have larger specific leaf area and foliar nutrient concentrations than noninvasive alien species (Grotkopp \& Rejmánek, 2007; Herron et al., 2007) and native species (Leishman et al., 2007; Peñuelas et al., 2010; van Kleunen et al., 2010b), traits conferring a 'fast growth' life history are typical of pioneer, disturbance-associated species with high light requirements for growth and survival (Wright et al.,2004). As a result, the role of fast growth rates and associated traits in determining invasion success is likely to be dependent on the habitat (Schumacher et al., 2008; Leishman et al., 2010) and on environmental conditions (Turnbull et al., 2008). A high potential relative growth rate under optimal conditions may allow alien species to take greater advantage of high resource availability when it arises, which would be consistent with the theory of fluctuating resources and community invasibility (Davies et al., 2004). One potential explanation for why faster-growing alien species are more globally widespread could be that anthropogenically disturbed and open habitats that suit fast-growing species the most may be more uniform and globally widespread than closed, undisturbed habitats.

Relative growth rates are known to decline as plants increase in size (West et al., 2001), meaning that measures of relative growth rate are essentially confounded by initial plant size (Rose et al., 2009). Thus, comparative studies considering the relation- ship between relative growth rate and invasiveness need to ensure that plants are of a similar ontogeny across species, or that relative growth rates are corrected for initial plant size (Rose et al., 2009). The dataset used here has the advantage that plant RGR was measured in the early phase of near exponential growth after germination, which should minimize the confounding effect of initial plant size on mean and maximum RGR measurements made (Grime \& Hunt, 1975). Ontogenetic variability in RGR may be responsible for inconsistencies among studies assessing the relationship between growth rates and invasiveness if studies measure growth rates at different ages. Measuring RGR at standardized stages of growth, characterization of growth rate curves or the use of alternative sizeindependent growth measures (Turnbull et al., 2008) could allow more valid comparisons among studies and species.

To conclude, we found support for the emerging consensus that alien plant invasiveness is often positively correlated with relative growth rate. We suggest that further investigation is needed to disentangle the effects of habitat context, introduction bias and global introduction effort on the general RGRinvasiveness relationship that is observed.

\section{ACKNOWLEDGEMENTS}

The authors thank the Swiss National Centre for Competence Research (NCCR) for NCCR Plant Survival project funding. The authors also thank Ewald Weber for use of his updated global invasive plant species list.

\section{REFERENCES}

Bailey, C.D., Koch, M.A., Mayer, M., Mummenhoff, K., O'Kane, S.L. Jr, Warwick, S.I., Windham, M.D. \& Al-Shebhaz, I.A. 
(2006) Toward a global phylogeny of the Brassicaceae. Molecular Biology and Evolution, 23, 2142-2160.

Bellingham, P.J., Duncan, R.P., Lee, W.G. \& Buxton, R.P. (2004) Seedling growth rate and survival do not predict invasiveness in naturalized woody plants in New Zealand. Oikos, 106, 308316.

Bolker, B.M. (2008) Ecological models and data in R. Princeton University Press, Princeton, NJ.

Bouchenak-Khelladi, Y., Salamin, N., Savolainen, V., Forest, F, van der Bank, M., Chase, M.W. \& Hodkinson, T.R. (2008) Large multi-gene phylogenetic trees of the grasses (Poaceae): progress towards complete tribal and generic level sampling. Molecular Phylogenetics and Evolution, 47, 488-505.

Davies, T.J., Barraclough, T.G., Chase, M.W., Soltis, P.S., Soltis, D.E. \& Savolainen, V. (2004) Darwin's abominable mystery: insights from a supertree of the angiosperms. Proceedings of the National Academy of Sciences USA, 101, 19041909.

Dietz, H. \& Edwards, P.). (2006) Recognition that causal processes change during plant invasion helps explain conflicts in evidence. Ecology, 87, 1359-1367.

Fior, S., Ola Karis, P., Casazza, G., Minuto, L. \& Sala, F. (2006) Molecular phylogeny of the Caryophyllaceae (Caryophyllales) inferred from chloroplast matK and nuclear rDNA ITS sequences. American Journal of Botany, 93, 399-411.

Goertzen, L.R., Cannone, J.J., Gutell, R.R. \& Jansen, R.K. (2003) ITS secondary structure derived from comparative analysis: implications for sequence alignment and phylogeny of the Asteraceae. Molecular Phylogenetics and Evolution, 29, 216234.

Gravuer, K., Sullivan, J.J., Williams, P.A. \& Duncan, R.P. (2008) Strong human association with plant invasion success for Trifolium introductions to New Zealand. Proceedings of the National Academy of Sciences USA, 105, 6344-6349.

Grime, J.P. \& Hunt, R. (1975) Relative growth rate: its range and adaptive significance in a local flora. Journal of Ecology, 63, $393-422$.

Grotkopp, E. \& Rejmánek, M. (2007) High seedling relative growth rate and specific leaf area are traits of invasive species: phylogenetically independent contrasts of woody angiosperms. American Journal of Botany, 94, 526-532.

Grotkopp, E., Rejmánek, M. \& Rost, T.L. (2002) Toward a causal explanation of plant invasiveness: seedling growth and lifehistory strategies of 29 pine (Pinus) species. The American Naturalist, 159, 396-419.

Hadfield, J. (2010) MCMC methods for multi-response generalized linear mixed models: the MCMCglmm R package. Journal of Statistical Software, 33, 2, 1-22.

Hamilton, M.A., Murray, B.R., Cadotte, M.W., Hose, G.C., Baker, A.C., Harris, C.J. \& Licari, D. (2005) Life-history correlates of plant invasiveness at regional and continental scales. Ecology Letters, 8, 1066-1074.

Hayes, K.R. \& Barry, S.C. (2008) Are there any consistent predictors of invasion success? Biological Invasions, 10, 483-506.

Herron, P.M., Martine, C.T., Latimer, A.M. \& Leicht-Young, S.A. (2007) Invasive plants and their ecological strategies: predic- tion and explanation of woody plant invasion in New England. Diversity and Distributions, 13, 633-644.

Hulme, P.E. (2005) Nursery crimes: agriculture as victim and perpetrator in the spread of invasive species. Crop science and technology, pp. 733-740. British Crop Protection Council, Alton, UK.

van Kleunen, M., Johnson, S.D. \& Fischer, M. (2007) Predicting naturalization of southern African Iridaceae in other regions. Journal of Applied Ecology, 44, 594-603.

van Kleunen, M., Weber, E. \& Fischer, M. (2010a) A metaanalysis of trait differences between invasive and non-invasive plant species. Ecology Letters, 13, 235-245.

van Kleunen, M., Dawson, W., Schlaepfer, D., Jeschke, J.M. \& Fischer, M. (2010b) Are invaders different? A conceptual framework of comparative approaches for assessing determinants of invasiveness. Ecology Letters, 13, 947-958.

Klotz, S., Kühn, I. \& Durka, W. (2002) BIOLFLOR-eine Datenbank zu biologisch-ökologischen Merkmalen der Gefaeßpflanzen in Deutschland. Bundesamt für Naturschutz, Bonn.

Lajeunesse, M.J. (2009) Meta-analysis and the comparative phylogenetic method. The American Naturalist, 174, 369-381.

Lamb Frye, A.S. \& Kron, K.A. (2003) rbcl Phylogeny and character evolution in Polygonaceae. Systematic Botany, 28, 326332.

Leishman, M.R., Haslehurst, T., Ares, A. \& Baruch, Z. (2007) Leaf trait relationships of native and invasive plants: community- and global-scale comparisons. New Phytologist, $176,635-643$.

Leishman, M.R., Thomson, V.P. \& Cooke, J. (2010) Native and exotic invasive plants have fundamentally similar carbon capture strategies. Journal of Ecology, 98, 28-42.

Liu, K., Eastwood, R.J., Flynn, S., Turner, R.M. \& Stuppy, W.H. (2008) Seed information database Available at: http:// www.kew.org/data/sid (release 7.1, accessed 20 May 2010).

Lockwood, J.L., Cassey, P. \& Blackburn, T.M. (2009) The more you introduce the more you get: the role of colonization pressure and propagule pressure in invasion ecology. Diversity and Distributions, 15, 904-910.

Martin, P.H., Canham, C.D. \& Marks, P.L. (2009) Why forests appear resistant to exotic plant invasions: intentional introductions, stand dynamics, and the role of shade tolerance. Frontiers in Ecology and the Environment, 7, 142-149.

Peñuelas, J., Sardans, J., Llusià, J., Owen, S.M., Carnicer, J., Giambelluca, T.S., Rezende, E.L., Waite, M. \& Niinemets, Ü. (2010) Faster returns on 'leaf economics' and different biogeochemical niche in invasive compared with native plant species. Global Change Biology, 16, 2171-2185.

Pyšek, P., Richardson, D.M., Pergl, J., Jarošik, V., Sixtovà, Z. \& Weber, E. (2008) Geographical and taxonomic biases in invasion ecology. Trends in Ecology and Evolution, 23, 237244.

Pyšek, P., Jarošik, V., Pergl, J., Randall, R., Chytrý, M., Kuhn, I., Tichý, L., Danihelka, J., Chrtek Jun, J. \& Sádlo, J. (2009) The global invasion success of Central European plants is related to distribution characteristics in their native range and species traits. Diversity and Distributions, 15, 891-903. 
$R$ Development Core Team (2009) $R$ : a language and environment for statistical computing. R Foundation for Statistical Computing, Vienna, Austria.

Randall, R. (2002) A global compendium of weeds. R.G. and F.J. Richardson, Meredith, Vic., Australia.

Rejmánek, M. (2005) Ecology of invasive plants: state of the art. Invasive alien species: a new synthesis (ed. by H.A. Mooney, R.N. Mack, J.A. McNeely, L.E. Neville, P.J. Schei and J.K. Waage), pp. 104-161. Island Press, Washington, DC.

Rejmánek, M. \& Richardson, D.M. (1996) What attributes make some plant species more invasive? Ecology, 77, 16551661.

Richardson, D.M. \& Rejmánek, M. (2004) Conifers as invasive aliens: a global survey and predictive framework. Diversity and Distributions, 10, 321-331.

Rose, K.E., Atkinson, R.L., Turnbull, L.A. \& Rees, M. (2009) The costs and benefits of fast living. Ecology Letters, 12, 1379-1384.

Schlaepfer, D.R., Glättli, M., Fischer, M. \& van Kleunen, M. (2010) A multi-species experiment in their native range indicates pre-adaptation of invasive alien plant species. New Phytologist, 185, 1087-1099.

Schumacher, E., Küffer, C., Tobler, M., Gmür, V., Edwards, P.J. \& Dietz, H. (2008) Influence of drought and shade on seedling growth of native and invasive trees in the Seychelles. Biotropica, 40, 543-549.

Spiegelhalter, D.J., Best, N.G., Carlin, B.P. \& van der Linde, A. (2002) Bayesian measures of model complexity and fit. Journal of the Royal Statistical Society B, 64, 583-639.

Stevens, P.F. (2001 onwards) Angiosperm phylogeny website. Available at: http://www.mobot.org/MOBOT/research/ APweb/ (accessed Version 9.1 March 2010).

Turnbull, L.A., Paul-Victor, C., Schmid, B. \& Purves, D.W. (2008) Growth rates, seed size, and physiology: do smallseeded species really grow faster? Ecology, 89, 1352-1363.

Webb, C.O. \& Donoghue, M.J. (2005) Phylomatic: tree assembly for applied phylogenetics. Molecular Ecology Notes, 5, 181183. Available at: http://www.phylodiversity.net/phylomatic (accessed 30 September 2009).

Webb, C.O., Ackerly, D.D. \& Kembel, S.W. (2008) Phylocom: software for the analysis of phylogenetic community structure and trait evolution. Bioinformatics, 24, 2098-2100.

Weber, E. (2003) Invasive plant species of the world. A reference guide to environmental weeds. CABI Publishing, Wallingford.

West, G.B., Brown, J.H. \& Enquist, B.J. (2001) A general model for ontogenic growth. Nature, 413, 628-631.

Wilkström, N., Savolainen, V. \& Chase, M.W. (2001) Evolution of the angiosperms: calibrating the family tree. Proceedings of the Royal Society B: Biological Sciences, 268, 2211-2220.
Wojciechowski, M.F., Lavin, M. \& Sanderson, M.J. (2004) A phylogeny of legumes (Leguminosae) based on analysis of the plastid matK gene resolves many well-supported subclades within the family. American Journal of Botany, 91, 1846-1862. Wright, I.J., Reich, P.B., Westoby, M. et al. (2004) The worldwide leaf economics spectrum. Nature, 428, 821-827.

\section{SUPPORTING INFORMATION}

Additional Supporting Information may be found in the online version of this article:

Appendix S1 Species and data from Grime \& Hunt (1975) and the Global Compendium of Weeds (Randall, 2002) included in this study.

Appendix S2 The phylogeny used in this study (in Newick format).

Appendix S3 Results from saturated models including interactions between life cycle and the number of Global Compendium of Weeds (GCW) references/global regions invaded.

As a service to our authors and readers, this journal provides supporting information supplied by the authors. Such materials are peer-reviewed and may be reorganized for online delivery, but are not copy-edited or typeset. Technical support issues arising from supporting information (other than missing files) should be addressed to the authors.

\section{BIOSKETCHES}

Wayne Dawson is a post-doctoral plant ecologist. His research focuses on the ecology of plant invasions in tropical and temperate ecosystems. He is also interested in the development and application of global weed risk assessment systems.

Markus Fischer is a professor in plant ecology, and is the leader of the plant ecology research group at the University of Bern. His interests mainly include conservation biology and plant population biology, biodiversity and ecosystem functioning, plant rarity and invasions.

Mark van Kleunen is a senior evolutionary plant ecologist. His research is currently focused on plant phenotypic plasticity and on determinants of native plant rarity and alien plant invasiveness. 